\title{
Historical Metamodels of Agency
}

Different agentic metamodels correspond to major historical periods of civilized humanity. Technological innovation is an important, distinguishing feature of this narrative (Spar, 2020). The long-term trend is toward greater agentic capability and potentiality, assisted by more sophisticated technologies: from the static agentic metamodels and simple technologies of premodernity to the more complex metamodels of modernity, assisted by mechanical and analogue technologies, and now to the increasingly dynamic, digitally augmented metamodels of the contemporary period. In summary, the evolution of agentic metamodels is a historical process itself. Each major period warrants detailed discussion.

\subsection{Major Historical Periods}

In premodern cultures-prior to the modern period of Enlightenment and industrialization-human agency was popularly conceived in terms of divinely ordained narratives and fixed social orders. Purposive thought and action were supervised by patriarchal authority and supernatural 
beings, which helped to make sense of intractable fate. For most people, the order of things was not a human composition, but bestowed by agents from above and beyond (Geertz, 2001). Given these assumptions, explanation of the world was teleological and driven by final cause, while categories of reality were defined in terms of essential states and forms. Reflecting this relative lack of capability and potentiality, the dominant metamodels of agency assumed stability (Sorabji, 2006). Normality meant replicating an established, often divinely ordained metamodel of agency, and significant variance was viewed as a sign of weakness or deviance. Hence, trying to amend or circumvent the divine order was fraught with existential risk, as the ancient Greek tragedians understood (Williams, 1993). And for most, human overcoming was not explained by autonomous reasoning and action, but by good fortune and supernatural beneficence.

In like fashion, explanatory thought about agency in premodernity focused on collective norms, compliance with them, and the vicissitudes of fate. Rare opportunities for change consisted in altering position within the established order, shifting from point to point, like transposing scalar values in Euclidean space (Isin, 2002). Not surprisingly, therefore, premodernity did not privilege individual agency, but rather deference and compliance. Granted, some scholars explored alternative conceptions, but as the trial of Socrates illustrates, encouraging autonomous critical thought could be a crime punishable by death (Hackforth, 1972). Agentic performance was assessed in terms of adherence to norms, and the purpose of feedback was to refine replication and correct deviation. Thomas à Kempis (1952) epitomized this perspective in The Imitation of Christ, one of the most revered texts of the premodern Christian period. He explains that fulfillment comes from absorbing scripture and imitating the life of Christ, not from autonomous reasoning and choice. The latter perspective had to wait for Martin Luther, who nailed his 95 theses to the door almost a century later. In summary, premodern, agentic metamodels prioritized replication, rather than adaptive change or original composition. 


\section{Replicative Metamodels of Agency}

Figure 2.1 depicts a premodern, replicative metamodel of agency. It builds on the cognitive-affective model of personality developed by Mischel and Shoda (1998). The figure assumes an input-process-output view of personality and the self, situated in context, but with relatively low variability and high stability. Hence, the metamodel illustrates a "persons in context" perspective, albeit with relatively low levels of contextual and system variation. Given these assumptions, the figure includes a sequence of major phases. First, it shows situational input stimuli (labeled SI), which include information about situations and problems in the world. Second, these stimulate sensory perception (SP), which transmits information to the next stage, cognitive-affective processing (CA). Third, the agent then processes information using cognitive-affective processing units (PU), which may include encodings, beliefs, affective states, goals, and values, including reference criteria and core commitments (RC), and self-regulatory schemes. Fourth, the agent generates action plans (AG), which are self-directed and self-regulated, to some degree. Fifth, these plans result in behavioral-performative outputs (BP). Sixth, such outputs trigger evaluation of performance (EP), as agents compare outcomes to aspirations and expectations, conditional on their degree of sensitivity to variance.

The figure also depicts three mechanisms of update encoding which flow from the evaluation of performance. These are shown by the arrowed

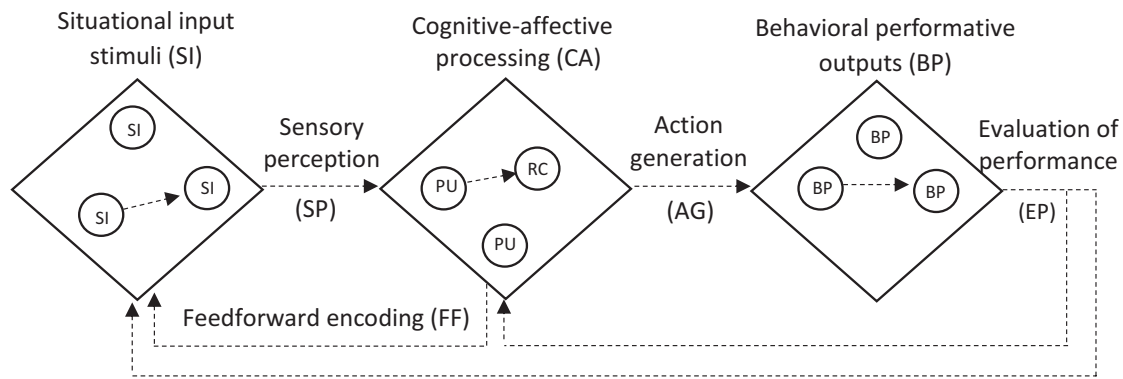

Feedback encoding (FB)

Fig. 2.1 Replicative agentic metamodel 
lines at the base of the figure. The first two are feedback mechanisms (FB) which flow from evaluation of performance (EP). One is an inter-cyclical mechanism which updates cognitive-affective processes; that is, updates occur at the completion of full cycles of processing. A second intercyclical mechanism updates input stimuli and the situational context itself. In addition, there is an intra-cyclical feedforward mechanism (FF), flowing from cognitive-affective processing, which updates the situation itself and input processing. It is intra-cyclical, in relative terms, because it occurs during and influences the ongoing process. Natural, human feedforward guidance is neurological and largely unconscious (Basso \& Belardinelli, 2006), and was inherently part of human functioning, even in premodern times.

Furthermore, each phase is composed of functional components, illustrated by small circles. These are situational inputs (SI), cognitive-affective processing units (PU), including one dotted circle showing a referential criterion or commitment (RC), and behavior performances (BP) (see Mischel \& Shoda, 1995). In the replicative metamodel, many components are invariant owing to stable contexts and behavioral norms. Arrows are dashed, to indicate relatively low variance and potentiality. Similarly, the figure depicts weak mechanisms of feedback and feedforward encoding, also shown by dashed lines. Premodern cultures did not exhibit widespread self-reflective variation, in this regard, but rather compliant imitation. Equally, learning was largely a process of memorization. As noted above, agents were guided by replication and imitative processes.

\section{The Modern Period}

By contrast, during the modern period, agentic capabilities and potentialities expanded, supported by technological innovation and socioeconomic development. For good and ill, new sources of knowledge, production, and mobility disrupted the premodern socioeconomic order. The focus of agency shifted away from patriarchal order and imagined beings, toward reasoning persons in the natural world (Giddens, 1991). Identity and meaning were now contingent on learning and achievement, rather than compliant acceptance of inherited position. That said, docility within social collectives remained hugely important, but it now 
became a political and philosophical question, versus one of theological dogma (e.g., Locke, 1967). In this fashion, modern criteria of reality, truth, and reasoning transcend premodern replication. Furthermore, the nature of human empathy and commitment frame modern thought about justice and ethics, rather than divine personalities and their pronouncements.

Consequently, the development of intelligent capabilities and the provision of opportunities for personal development and learning have been central to modern human science and theories of agency. Scholars examined the functioning of mind and personality, and the potential impact of social and cultural forces on human development (Pinker, 2010). Educational and clinical interventions built on such research. In parallel, the modern sciences became deeply dualistic. As noted in Chap. 1, for many scholars, human mind and consciousness were distinguished from material nature and the body. Hence, the human and natural sciences bifurcated into separate systems of study, with different methods of observation and analysis. Most critically, while the fundamental realities of mind and self may be directly accessible to ordinary consciousness and intuition, understanding of the natural world demands specialist technologies in controlled settings.

Reaction to Darwin's theory of evolution epitomizes this divide (see Mayr, 2002). On the one hand, the biological world was reconceived as a fully natural system, requiring scientific methods of observation and analysis, not driven by essentialism or teleology. On the other hand, however, many continued to believe that the fundamental features of mind and self were accessible to ordinary consciousness and irreducible to natural cause. Indeed, they feared that natural mechanisms would erode the ontological status of self-consciousness, and with it, various precepts of identity and faith. Subsequent debates reflect this dualism of modern thought: how to reconcile and integrate material cause and natural evolutionary mechanisms, with human consciousness, intentional action, and the interpretation of meaning.

Modern agentic metamodels exhibit the same tension. Most are deeply dualistic and assume problematic relations between the material and conscious aspects of human experience, or in other words, between mind and body. Reflecting this dualism, the major problems of modern agency can be compared to opposing vectors in Cartesian space: material versus 
intentional cause; natural selection versus preferential choice; biological instinct versus autonomous will (Reill, 2005). These polarities combine and often clash, in metamodels of evolutionary change and adaptive learning. Modern human science then seeks to resolve the resulting dilemmas. It asks, how do biological evolution and development interact with conscious mind and learning? Nevertheless, both mental and material processes involve change and development, albeit via different mechanisms. In consequence, the dominant agentic metamodels of modernity are broadly adaptive, rather than replicative.

\section{Modern Adaptive Metamodels}

Modern adaptive metamodels of agency therefore assume autonomous, reasoned problem-solving, learning, and development, within natural and cultural worlds. Persons are generally described as complex, open, adaptive systems, embedded in context (Shoda et al., 2002). Figure 2.2 illustrates this type of adaptive metamodel. Once again, it includes the same broad phases: situational input stimuli (SI) trigger sensory perception (SP), which in turn stimulate cognitive-affective processes (CA) by interacting processing units (PU), including one dotted circle showing a referential criterion or commitment (RC). These processes lead to action generation (AG) and resulting behavioral-performative outputs (BP), and the evaluation of performance (EP), which results in feedforward and feedback encoding (FF and FB respectively). Importantly, the modern, adaptive metamodel assumes stronger capabilities and more advanced technologies, when compared to the premodern, replicative metamodel in Fig. 2.1.

The figure depicts other important changes, compared to the replicative metamodel. To begin with, the metamodel in Fig. 2.2 is more complex, shown by additional component circles in each segment. The system is also more connected and dynamic, shown by the greater number of arrows, which are now solid rather than dashed, indicating stronger capabilities and potentialities. Hence, Fig. 2.2 shows greater functional intensity overall. Particularly, cognitive-affective processing is a more complex 


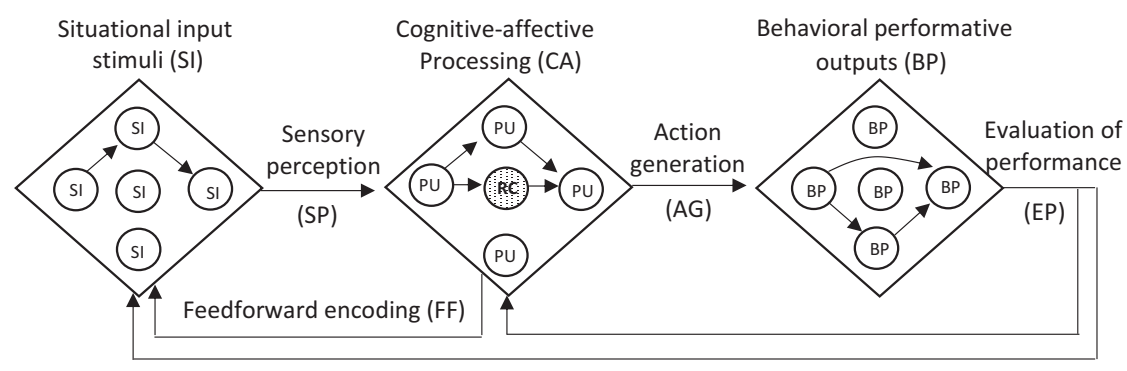

Feedback encoding (FB)

Fig. 2.2 Adaptive agentic metamodel

system of interacting units. Some of these units—especially beliefs and values_-will also serve as reference criteria and core commitments, which guide action and the evaluation of outcomes. One such criterion or commitment is depicted by a dotted circle (RC). In actual systems, there will be many.

In addition, the adaptive metamodel in Fig. 2.2 has stronger feedback and feedforward mechanisms, indicated by the solid arrowed lines at the base of the figure. These solid lines represent the fact that variance often triggers adaptive learning in modern contexts, as well as updates to the stimulus environment and the system itself. Modern agents therefore exhibit stronger reflexive functioning, compared to agents in premodernity. Inter-cyclical feedback (FB) is more active as well. Moreover, some updates will amend reference criteria and core commitments. Although, scholars continue to debate which reference criteria and commitments are adaptive, when, why, and to what degree.

\section{The Period of Digital Augmentation}

In response to digitalization, agentic metamodels are transforming again. The technological assistance of agency is transitioning to a new level of scale, speed, and sophistication, thus driving a qualitative shift in agentic capability and potentiality. To begin with, recall that advanced artificial agents can compose, decompose, and recompose metamodels, in a 
dynamic fashion, potentially self-generating without external supervision. In addition, they learn with extraordinary speed and precision, including from intra-cyclical feedforward updates. This latter capability is particularly important. Earlier metamodels assume modest feedforward mechanisms, either from unconscious instinct or the effortful guidance of complex processes over time, whereas digitally augmented agents will learn rapidly and constantly in this fashion. As mentioned previously, advanced artificial agents already do. When incorporated into humanartificial collaboration, therefore, rapid feedforward learning, plus the sheer power and reach of artificial agency, transform agentic functioning. Augmented agency is intensely generative and near composability. Indeed, these capabilities distinguish digitalization from earlier periods of technologically assisted agency.

All aspects of processing are affected. Augmented agents can sense and sample the world more extensively, organize and process vast amounts of information very rapidly, represent and solve complex problems, and then design and direct responsive action. Augmented agents also achieve unprecedented speed and precision in learning, compared to purely human agents. To illustrate, consider the artificial agency required for autonomous mobility systems: constant real-time sensing of the environment, vehicles, and passengers; rapid complex problem-solving and empathetic interaction; accurate and coordinated action planning and control. Experts therefore apply a framework for autonomous vehicles known as "sense, plan and act" (Shalev-Shwartz et al., 2017). These systems exemplify that generative, augmented agency constitutes a new metamodel of intelligent agency (see Caro et al., 2014). Its central characteristics include the following: close collaboration between human and artificial agents; high sensitivity to context; intelligent sampling, representation, and resolution of complex problems; compositive methods, based in artificial intelligence; high sensitivity to variance and rapid evaluation of performance; very rapid processing and learning rates; real-time monitoring, self-regulation, and adjustment; often self-generative with minimal external supervision.

This historic transformation has meaningful topographical analogues in formal modeling. First, digital augmentation far transcends the state changes of the premodern period, which are comparable to scalar 
transpositions, shifting from point to point in Euclidean space. Second, digitalization also transcends the adaptive learning of modernity, which can be mapped as vector transitions in Cartesian space. Now third, and in contrast to both earlier periods, digital augmentation is about generative composition, which can be expressed as multi-vector tensor transformations, curving through Riemannian space (Kaul \& Lall, 2019).

\section{Generative Metamodels}

Figure 2.3 illustrates this kind of generative metamodel of agency. Once again, the system integrates situational input stimuli (SI) which trigger sensory perception (SP), which in turn stimulate cognitive-affective processes (CA) consisting of interacting processing units (PU), again including one dotted circle showing a referential criterion or commitment (RC). As before, processing results in action generation (AG) leading to behavioral-performative outputs (BP), and the evaluation of performance processing and outcomes (EP), which often results in feedforward and feedback encoding (FF and FB respectively). However, this metamodel is more complex and dynamic, compared to the metamodels depicted in Figs. 2.1 and 2.2. This is shown by the greater number of component

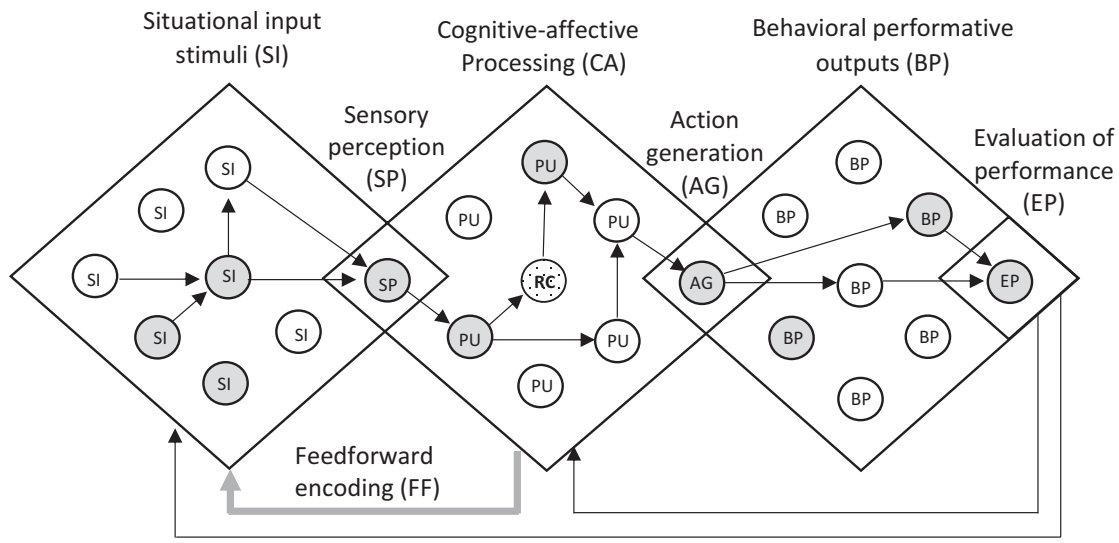

Feedback encoding (FB)

Fig. 2.3 Generative agentic metamodel 
circles within each segment. Some are now shaded as well, which indicates they are digitalized, transformed by the incorporation of digital technology. Importantly, the major phases shown by large diamond shapes now partially overlap, integrated by digitalized processes.

As Fig. 2.3 further shows, digitalization occurs throughout the metamodel. First, the generation of situational inputs is increasingly digitalized. For example, through the internet of things and intelligent sensors, situational contexts are increasingly digitalized and connected. In consequence of this development, the figure also shows that sensory perception overlaps with cognitive-affective processing. Both phases are digitally intermediated. Second, cognitive-affective processing is equally digitalized and collaborative, whereby artificial agents interact with the cognitive-affective system. In this regard, recent innovations include cognitive computing, wearable devices, and artificial personality (Mehta et al., 2019). Reflecting this development, the figure shows that action generation is digitalized and overlaps cognitive-affective processing and behavioral-performative outputs. That is, digitalized action generation intermediates cognitive-affective processing and behavior performance. Current examples of this include artificial assistants and expert decision support systems (Wykowska, 2021). Third, behavioral-performative outputs are themselves digitalized, for example, by the incorporation of artificial agents, collaborative robotics, and intelligent prosthetics (Vilela \& Hochberg, 2020). Finally, the figure shows that evaluation of performance is partially digitalized too.

In summary, Fig. 2.3 shows how digitalization is augmenting and transforming all aspects of agency: the stimulus environment and perception of it; processes of reasoning and affect; the generation and performance of self-regulated action; the evaluation of performance, and the encoding of updates as learning. For this reason, the figure includes a stronger, digitalized stream of feedforward encoding (FF), flowing from cognitive-affective processing to update the situational context and processing itself. Now depicted by a heavier shaded line, indicating it is digitalized. Via this process, the system updates the context and process itself, intra-cyclically, in real time. Today's most advanced agents already function in this way. Newer technologies, including devices which integrate real-time biometric feedback and augmented reality, will accelerate this 
trend. Feedforward updating will be constant and ubiquitous. In consequence, augmented agents will be increasingly self-generative, far eclipsing the agentic potentiality of earlier periods. These will be distinguishing features of generative, agentic metamodels.

At the same time, however, owing to their complexity and dynamics, these metamodels will be more difficult to supervise. Artificial and human agents will interact in every phase and function. However, as the previous chapter explains, both agents function in different ways. Much human processing is relatively myopic, sluggish, layered, and approximating, while artificial agents are increasingly fast, expansive, compressed, and precise. In consequence, many artificial feedforward mechanisms are inaccessible to human consciousness, and hence the two levels of processing could easily diverge. If this happens, augmented agents risk dysfunctional combinations of precision and approximation, fast and slow processing rates, sensitivity and insensitivity to variance, layering and compression, and complexity plus simplification. Artificial agency could then outrun, overwhelm, and bypass human inputs. Alternatively, human myopia and bias may infect artificial agents, and digitalization would then reinforce and amplify the limitations of human functioning. In summary, digitally augmented, generative metamodels pose major supervisory challenges.

\subsection{Agentic Activation Mechanisms}

Agentic activation mechanisms are being digitally transformed as well. To begin with, consider Fig. 2.2 once again, which shows the modern, adaptive metamodel of agency. All components are clearly distinguished and bounded. They are exogenous (external) or endogenous (internal), relative to each other. For example, situational inputs (SI) are exogenous to cognitive-affective processing (CA), whereas processing units (PU) are endogenous to cognitive-affective processing (CA). Now compare the digitally augmented, generative metamodel in Fig. 2.3. The figure is more highly integrated, with digitalized components connecting the major stages of situational inputs (SI), cognitive-affective processing (CA), and behavior performances (BP). These stages now overlap, owing to the 
digitalization of activation and intra-cyclical feedforward mechanisms (FF). Via such means, augmented agents will update the system in real time (Ojha et al., 2017). These mechanisms are central to the generative metamodel of agency.

Regarding the first two stages, digitalized mechanisms of sensory perception (SP), join the two large diamond shapes of situational inputs (SI) and cognitive-affective processes (CA). As a result, sensory perception becomes an intelligent process itself, thanks to the rapid intra-cyclical management of attention and sampling (see Fiedler \& Wanke, 2009). In fact, environmental sampling and data gathering become deliberate, intelligent, and adaptive activities. Recent evidence supports this shift (e.g., Dong et al., 2020). The internet of things, smart sensors, wearable devices, and automated systems of multiple kinds, all connected to artificial agents, enable intelligent sensing and sampling, which radically complement ordinary sensory perception. However, from a modeling perspective, the digitalized mediation of intelligent sensory perception (SP) is neither exogenous nor endogenous, with respect to situational inputs (SI) and cognitive-affective processing (CA). Rather, intelligent sensory perception is in-between, mediating the boundaries of both the situational context and the cognitive-affective system.

Second, in like fashion, Fig. 2.3 shows that action generation (AG) is becoming behavioral and performative, not simply an antecedent of behavior. Action generation is now digitalized and joins cognitiveaffective processing (CA) and behavior performance (BP). This means that action plans can be updated and regenerated during performances, in real time, via intra-cyclical feedforward mechanisms (Heaven, 2020). Artificial agents will process rapidly in the background, to integrate and update each phase of the process. Performances thus become more dynamic, thanks to digitalization. Hence, we can refer to performative action generation in generative metamodels. In fact, this already occurs in the development of agile, self-correcting systems (Howell, 2019). However, performative action generation $(A G)$ is neither exogenous nor endogenous, with respect to cognitive-affective processing (CA) and behavior performance $(\mathrm{BP})$. Rather, the process is again in-between, mediating the boundaries of both the cognitive-affective system and behavior performance. 
Third, Fig. 2.3 shows that situational updating from feedback (FB) and feedforward (FF) is becoming intelligent itself. That is, situational contexts are becoming sites of intelligent learning, not simply passive sources of sensory inputs and problems. Once again, enabling technologies include the internet of things, ambient computing, and autonomous agents. All are embedded into the problem context and capable of updating it, often autonomously, in a self-generative fashion. Via these mechanisms, contexts will update and regenerate during problem-solving, not only from inter-cyclical, adaptive feedback. Hence, existing situations will evolve, and new ones emerge, during problem-solving itself. I describe this process as contextual learning, which is neither exogenous nor endogenous, with respect to behavior performance and the stimulus environment. Rather, the process is also in-between, mediating the boundaries of both the evaluation of performance (EP) and situational inputs (SI).

\section{Augmented In-Betweenness}

All the mediating mechanisms just described are central to augmented agency: intelligent sensory perception, performative action generation, and contextual learning. They are novel and transforming. Together, they allow augmented agents to learn, compose, and recompose in a dynamic fashion, updating form and function in real time. Augmented agency is therefore near composability, not only near decomposability. Moreover, these mechanisms signal a wider shift, from fixed boundaries and categories to fluid metamodeling. In consequence, however, being inside or outside of system boundaries at any time (endogenous or exogenous respectively) is often ambiguous and may not apply. This is because digitalized mediators operate at a higher rate and level of sensitivity, monitoring and adjusting system boundaries (see Baldwin, 2018). They are neither endogenous nor exogenous, relative to the boundaries they help to define. Rather, they are consistently in-between, processing potential form and function. These mechanisms will be critical and ubiquitous within digitally augmented agents.

Over recent years, scholars pay increasing attention to such effects. This interest is captured by the growing number of studies about forms of 


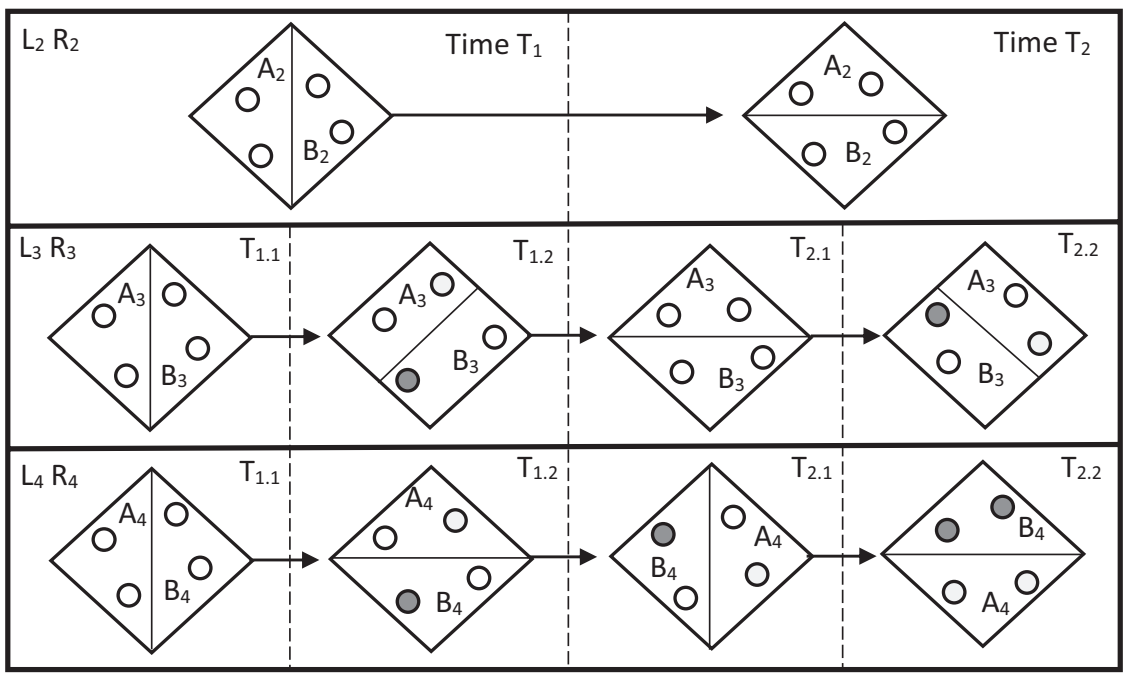

Fig. 2.4 Endogenous, exogenous, and in-between

ambiguous meaning, ambivalent value and belief, organizational hybridity, and ambidextrous action (e.g., March, 2010; O'Reilly \& Tushman, 2013). In many domains, agents combine alternative, complementary, and sometimes conflicting patterns of thought and action, as they grapple with increasing phenomenal complexity and dynamism. This leads to shifting categorical boundaries, forms, and functions. The prefix "ambi," meaning both in Latin, is therefore a recurrent prefix in descriptive terms. As the reader will see in subsequent chapters, I exploit this prefix to describe other, novel patterns of in-betweenness arising from digitalization.

Figure 2.4 illustrates this type of dynamic mediation. It shows three levels and rates of processing and highlights the potential for divergence within augmented agents. To begin with, the upper third of the figure is modern adaptive capabilities at level $\mathrm{L}_{2}$. They cycle at rate $\mathrm{R}_{2}$ over times $\mathrm{T}_{1}$ and $T_{2}$, and process inter-cyclical feedback at $T_{2}$. The middle third of the figure is stronger digitalized capabilities at level $\mathrm{L}_{3}$ which cycle at rate $\mathrm{R}_{3}$ over times $T_{1.1}, T_{1.2}, T_{2.1}$, and $T_{2.2}$. In other words, digitalized processing $\mathrm{L}_{3} \mathrm{R}_{3}$ cycles more rapidly, compared to the modern adaptive scenario $\mathrm{L}_{2} \mathrm{R}_{2}$. The lower third of the figure illustrates digitalized capabilities at level $\mathrm{L}_{4}$ 
with cycle at rate $R_{4}$. These are roughly equal to $L_{3} R_{3}$ but are labeled differently to distinguish them. All three processes then depict the same two subsystems labeled A and B and their components. For example, these could be complementary subsystems of a problem-solving process.

However, the three levels of processing produce different patterns, shown by shaded dots and shifting boundaries of A and B. First, consider the upper portion of the figure, depicting $\mathrm{L}_{2} \mathrm{R}_{2}$. It shows that some of the components of $A_{2}$ and $B_{2}$ transition between times $T_{1}$ and $T_{2}$, and hence the boundary line shifts from being horizontal to vertical. For example, perhaps some components of solution search at $T_{1}$ become aspects of problem representation at $T_{2}$, reflecting adaptive learning which improves attention and problem sampling (Fiedler \& Juslin, 2006). Now compare the middle process at $\mathrm{L}_{3} \mathrm{R}_{3}$. Components and boundaries shift as well, but in a different fashion. Most notably, the process cycles more rapidly, and as a result, the system changes at time $T_{1.2}$. One component of each subsystem has moved, along with the system boundary. The light gray dot shows a component at $T_{1.2}$ which is endogenous to $A_{3}$ and exogenous to $B_{3}$, but it remains endogenous to $B_{2}$. While the dark gray dot shows a component at $T_{1.2}$ which is endogenous to $B_{3}$ and exogenous to $A_{3}$, but it remains endogenous to $A_{2}$. Importantly, if we now combine the two subsystems $\left(\mathrm{L}_{2} \mathrm{R}_{2}\right.$ and $\left.\mathrm{L}_{3} \mathrm{R}_{3}\right)$ in one augmented agent, some components are regularly in-between, simultaneously endogenous and exogenous, depending on the level of processing.

Note that at time $T_{2}$, both $\mathrm{L}_{2} \mathrm{R}_{2}$ and $\mathrm{L}_{3} \mathrm{R}_{3}$ are equivalent again. This could mean that the change at $T_{1.2}$ has now been incorporated into the system $\mathrm{L}_{2} \mathrm{R}_{2}$ via adaptive feedback. The same pattern of processing then occurs over the following cycle $\mathrm{T}_{2}$. Once again, some shaded components are in-between, simultaneously endogenous and exogenous, relative to different levels of processing. But the degree of divergence is modest, only one component of each subsystem at a time. Moreover, $\mathrm{L}_{2} \mathrm{R}_{2}$ and $\mathrm{L}_{3} \mathrm{R}_{3}$ will synchronize at the completion of each major cycle; although inbetween, they exhibit ambiguous boundary conditions. In summary, this augmented agent is broadly convergent over time, because digitalized intra-cyclical, feedforward updates at $L_{3} R_{3}$ are incorporated into $L_{2} R_{2}$ via inter-cyclical adaptive feedback. The agent absorbs updates effectively at both levels. Learning is generative and functional, in these respects. 
Next, consider the third process at $\mathrm{L}_{4} \mathrm{R}_{4}$. Once again, components and boundaries shift, but now more extensively, compared to the other processes. At time $\mathrm{T}_{1.2}$ components of $\mathrm{A}_{4}$ and $\mathrm{B}_{4}$ have moved, and the boundary between them is now vertical. Once again, the light gray dot shows a component at $T_{1.2}$ which is endogenous to $A_{4}$, but it remains endogenous to $B_{2}$. While the dark gray dot at $T_{1.2}$ is endogenous to $B_{4}$, but it remains endogenous to $A_{2}$. Moreover, if we combine the two subsystems $\left(L_{2} R_{2}\right.$ and $\left.L_{4} R_{4}\right)$ in one augmented agent, half the components are in-between, simultaneously endogenous and exogenous, relative to different levels of processing.

Furthermore, at time $T_{2}$, the two processes $L_{2} R_{2}$ and $L_{4} R_{4}$ remain divergent, in contrast to the earlier convergent condition. Shaded components are persistently in-between, ambiguously endogenous and exogenous. In other words, the change in $\mathrm{L}_{4} \mathrm{R}_{4}$ at $\mathrm{T}_{1.2}$ is not fully incorporated into the system $\mathrm{L}_{2} \mathrm{R}_{2}$, probably because the degree of digitalized processing at $\mathrm{L}_{4} \mathrm{R}_{4}$ is beyond the absorptive capabilities of $\mathrm{L}_{2} \mathrm{R}_{2}$. Moreover, the same pattern of divergent processing occurs again over the following cycle $\mathrm{T}_{2}$. It leads to a compounding effect. By time $T_{2.2}$, all components of $A$ and $B$ are ambiguously endogenous and exogenous. This augmented agent is therefore increasingly divergent over time because digitalized intra-cyclical, feedforward updates at $\mathrm{L}_{4} \mathrm{R}_{4}$ are not incorporated into $\mathrm{L}_{2} \mathrm{R}_{2}$ via inter-cyclical adaptive feedback. The agent does not absorb updates effectively across levels and modalities. Learning is digitalized but dysfunctional, in these respects.

In fact, actual systems already exhibit these effects (e.g., Lee \& Ro, 2015), for example, in the dynamic adaptation of modular transaction networks and software architecture (Baldwin, 2008). These processes rapidly update modular components, setting and resetting system boundaries. However, challenges escalate when inter-cyclical feedforward processing is fast and constant. Boundaries are consistently in flux. Hence, in highly digitalized systems, there will always be some components which are in-between, ambiguously endogenous and exogenous. The risk is that rapid, intra-cyclical updates will lack coordination with slower, inter-cyclical feedback, as depicted in Fig. 2.4. When this occurs in augmented agents, artificial and human processes will diverge, possibly leading to dysfunctional outcomes. Effective supervision will be critical. 


\section{Entrogenous In-Betweenness}

Standard concepts fail to capture these novel features of digitalization. Indeed, most human sciences view in-betweenness as transitional, temporary, or paradoxical. The closest concept is liminality, but even it implies being ephemeral, and permanent liminality is viewed as dysfunctional, a sign of faulty, incomplete processing (Ibarra \& Obodaru, 2016). Therefore, to capture this novel type of ongoing in-betweenness, another term will be helpful. I propose "entrogenous" which builds on "entre," meaning between in numerous European languages. Applied to the generative metamodel of agency, "entrogenous" and "entrogeneity" refer to the digitalized mechanisms which mediate in-betweenness, and whereby forms and functions develop and transform. Notably, such mechanisms are neither endogenous nor exogenous, relative to fixed boundaries. Rather, they are constantly in-between and mediating potential boundaries. The major risk, as shown by Fig. 2.4, is that poor supervision of entrogenous mechanisms will lead to divergent processes and dysfunctional outcomes. This particularly applies to the novel, digitalized mediators of augmented agency identified earlier: intelligent sensory perception, performative action generation, and contextual learning.

In fact, this puzzle is far from new. In ancient Greece, Heraclitus famously wrote, "You cannot step into the same river twice, for other waters are continually flowing on." Equally important but less well known, he also wrote, "We step and do not step in the same rivers. We are and are not" (Kirk, 1954). In other words, human experience, thought, and action are inherently in-between, constantly in flux. Form and function are relative to the frame of reference. As Heraclitus observed, a river is defined by its banks and flowing waters, and therefore simultaneously stable and always changing. In-betweenness is then normal, not dysfunctional. This Heraclitian perspective contrasted the thought of Plato and Aristotle, who favored categorical stability and essential order. Digitalized agentic systems address this ancient dilemma, because they allow for continual composition and recomposition at multiple levels. Hyperparameters and parameters are set and reset, as processes unfold (Feurer \& Hutter, 2019). With respect to augmented agency, form and function will stabilize for a time, depending on the context, and 
recompose as contexts change. This is the core dynamic of generative, augmented agency. By analogy, therefore, entrogenous mediation is Heraclitian rather than Aristotelian. The implication being, that any instance of perceived permanence is mediated by some process in constant flux. To cite Herbert Simon (1996) again, science often transforms assumed states into dynamic processes.

At the same time, human and artificial agents possess different, inherent capabilities and potentialities. Much human processing is relatively sluggish, parochial, and heuristic, while artificial agents are increasingly fast, expansive, and precise. Therefore, what is entrogenous for artificial agents, may appear exogenous or endogenous for humans. As Heraclitus wrote, "We are and are not." These entrogenous dilemmas amplify the risks identified previously. Human agents could import inflexible categories, beliefs, and biases into augmented agency. Endogeneity and exogeneity would be baked in, from a human perspective. At the same time, however, artificial agents could relax categorical boundaries, and allow for greater plasticity and variation. The overall result will be divergent, and potentially conflicting, agentic form and function. In fact, these dilemmas are already observed in semi-supervised, collaborative systems (Kouvaris et al., 2015). They will be even more pronounced in larger, augmented communities and organizations. Later chapters will revisit this issue in relation to specific functional domains.

\section{Summary of Metamodels}

We can now summarize the foregoing discussion. To begin with, there are major differences between human and artificial agents. Natural human agents tend toward stability and possess limited capabilities and potentialities. As a result, purely human metamodels are relatively stable, weakly assisted by technologies, and not highly adaptive. They often possess deeply encoded hyperparameters, parameters, and variables, and fixed boundary conditions. Figure 2.1 illustrates this type of system labeled the replicative, agentic metamodel, which was dominant during premodernity. Next, as capabilities and technological assistance advanced, human agents became more autonomous and developmental, as shown in the 
modern, adaptive metamodel in Fig. 2.2. Granted, significant limits remain. Nevertheless, the adaptive metamodel of modernity affords greater degrees of freedom, compared to replicative metamodels. Boundaries are more adaptive and less fixed.

Digital augmentation now promises far greater capabilities and potentialities. Most notably, digitalization enables augmented agents which combine human and artificial agents in close collaboration. A major feature will be the capability for generative metamodeling, effectively in real time. Digitalized entrogeneity will be fundamental, mediating the dynamic composition of agentic form and functioning. This type of digitally augmented, generative metamodel is shown in Fig. 2.3. If well supervised, augmented agency and humanity will enjoy greater degrees of freedom and potentiality. But there are also major risks and dilemmas to resolve.

\subsection{Dilemmas of Digital Augmentation}

The greatest benefits of digital augmentation are its potential weaknesses. As often happens, remarkable strengths easily skew performance outcomes. On the one hand, augmented agents will sample and search ever more widely, process information at increasing speed, scale, and accuracy, and learn at unprecedented rates. On the other hand, thanks to human semi-supervision, augmented agents will often inherit myopias, biases, and parochial commitments. Therefore, digitally augmented agency confronts a fundamental challenge: how to combine and supervise human and artificial capabilities while avoiding excessive divergence, convergence, and distortion? Resolving these questions will be critical for augmented humanity.

\section{Problematics and Metamodels}

To clarify these topics further, Fig. 2.5 summarizes the agentic metamodels and problematics already discussed. It shows three agents X, Y, and Z, over three successive time periods labeled 1,2, and 3. The figure captures 
the essence of three broad historical periods, premodernity, modernity, and contemporary digitalization. First, recall that in premodern contexts, metamodels of agency assume low complexity, relatively poor capabilities and potentialities, with little variation. Agency tends to be imitative and replicative. Overall agentic functioning is viewed as a collective accomplishment, rather than an outcome of autonomous individuals. Predictably, therefore, premodern problematics focus on the integration of persons within communal narratives, and how to account for variation in a world of ordained stability and order (Walker, 2000). In Fig. 2.5, these conditions are shown by the segments with only one dot in each, which exclude agent $\mathrm{X}$ at time 3. Assume that each dot represents components of some agentic function. As the figure shows, functioning is widely dispersed across the collective (agents X, Y, and Z) over time (periods 1, 2, and 3). Each individual agent is weakly responsible for overall functioning at any time, apart from agent $\mathrm{X}$ at time 3 . Hence, they are highly dependent on each other. Therefore, if we assume that all the segments with one dot are required to perform a particular agentic process, then efficacious action will require the cooperation of all three agents over the three time periods, and hence the minimization of individual variance. In this way, the segments with one dot expose core features of the premodern, replicative metamodel and its associated problematics. Individuals must conform and cooperate over time, to achieve collective outcomes.

Notably, simple models of artificial intelligence and machine learning possess similar features. They, too, reference encoded models to solve predictable types of problems (Norvig \& Russell, 2010). Moreover, these simpler model-based, artificial agents, are frequently embedded within processing networks, just like the agents in Fig. 2.5 with only one major functional role. Functioning is therefore highly distributed. In these respects, simpler types of artificial agent exhibit metamodels which are comparable to those of premodern agency. Such technologies are therefore less genuinely "agentic" and augmenting, and sit at the passive end of the supervisory spectrum. That said, this suggests a promising avenue for research into replicative metamodels. Simpler model-based agents appear well suited to the task.

By contrast, during modernity, human agents develop stronger capabilities for autonomous thought and action. Agentic functioning is both 
an individual and collective accomplishment, and numerous technological innovations assist these developments. Modern problematics therefore focus on the reconciliation of individual freedom and collective solidarity, and the means by which humans adapt and transcend their limited capabilities and potentialities (Pinker, 2018). These problematics are illustrated by agent $\mathrm{X}$ and time 3, excluding the more densely dotted segment at the base of the figure. Notably, there are now extra dots within agent $\mathrm{X}$ at time 3, which shows that this individual is more capable and performs more functions, thanks in part to increased technological assistance. Indeed, agent X contains as many functional components at time 3 , as all other agents, which illustrates the agent's capability for autonomous action. Nevertheless, agent $\mathrm{X}$ remains reliant on the collective. Significant functions are still distributed, and effective performance will require cooperation with other agents over time. In these respects, agent $\mathrm{X}$ at time 3 illustrates core aspects of the adaptive metamodel and problematics of modernity, namely, how to develop individual capability and potentiality, while integrating with collective form and function?

Once again, there are strong parallels to artificial agents. In fact, the modern adaptive metamodel corresponds to goal-based and utility-based, artificial agents, which are more advanced than the simpler model-based agents discussed above (Norvig \& Russell, 2010). First, goal-based artificial agents use encoded preferences to guide problem-solving. Other things being equal, they seek to achieve predetermined outcomes. Second, utility-based artificial agents possess additional rules for the rank ordering of potential outcomes and then seek to maximize utility. Clear parallels exist in modern social and behavioral theories. In many such disciplines, agents are conceived as goal seeking, maximizing preferences and utility (Bandura, 2007; Thaler, 2016). Hence, the architecture of goal-based and utility-based artificial agents, is broadly comparable to the adaptive metamodel of modernity. Both entail intelligent agents, working in concert, seeking to achieve goals and maximize preferences.

Contemporary digitalization supports a new, generative metamodel of agency. It assumes high levels of complexity, unprecedented processing capability, and intense patterns of functioning at every level. Figure 2.5 also illustrates the core features of such a metamodel, by showing one component of agent $\mathrm{X}$ at the base of time 3, which is very dense with 


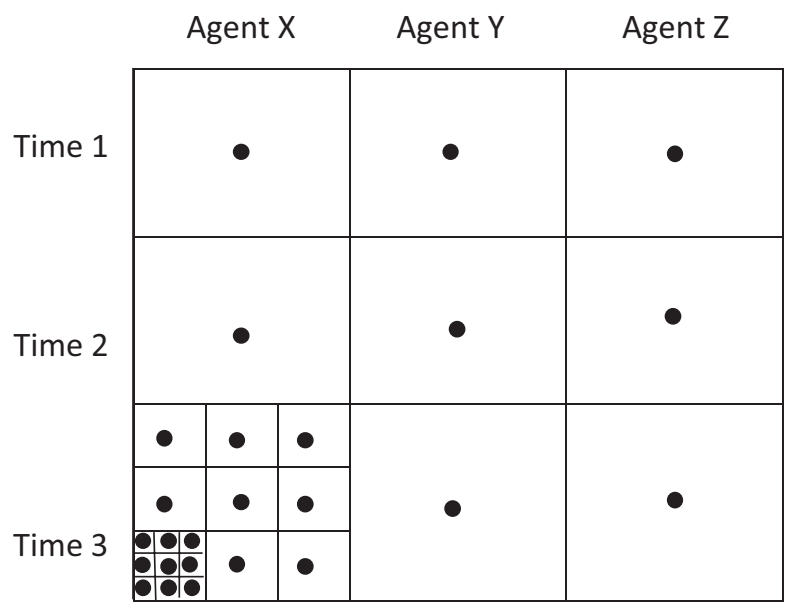

Fig. 2.5 Historical problematics of agency

dots. This component is fully digitalized. Indeed, this digitalized component of agent $\mathrm{X}$ exhibits as many functions as all other segments of $\mathrm{X}$, as well as the other agents in the figure. In a fully digitalized collective, all agents and components will be equally intense. The increase in functional complexity is exponential, across multiple levels and modalities, and within any time period as well. New problematics thus emerge: how can human beings collaborate closely with artificial agents, while remaining genuinely autonomous, in reasoning, belief, and choice; how will human and artificial agents learn to understand, trust, and respect each other, despite their different capabilities and potentialities; and how will augmented agents supervise the dynamic composition and recomposition of metamodels? And not surprisingly, this generative metamodel mirrors the architecture of the most advanced artificial agents, because it assumes participation by such systems. Advanced artificial agents will be integral to augmented agency.

In summary, the different functional patterns in Fig. 2.5 capture the history of both artificial and human agency. The figure shows how the recent evolution of artificial agency shares important features with the long history of human agency, at least in terms of their metamodels. First, model-based artificial agents map to the replicative metamodels of 
premodernity. Second, goal-based and utility-based, artificial agents mirror the adaptive metamodels of modernity. And third, advanced artificial agents instantiate the generative metamodels of digital augmentation. In these respects, the rapid ontogeny of artificial agency over recent decades, recapitulates the slow phylogeny of civilized humanity over millennia (see Clune et al., 2012). Or to paraphrase Hegel (1980), the recent history of digital science recapitulates the digitalized science of history. More striking still, both processes converge in the science of augmented agency because the story of digital science mirrors the science of augmented humanity. Producing a historical synthesis Hegel would surely appreciate.

\subsection{Patterns of Supervision}

Even in a highly digitalized world, however, people will continue to exhibit models of agency which are effectively premodern, in terms of their core components, levels of complexity, and modes of supervision. There will be a spectrum of artificial augmentation. In some contexts, that is, agency will still be governed and supervised in terms of replication and narrative, as in many cultural and faith communities. Similarly, people will continue choosing modern adaptive metamodels which entail less intrusive technological assistance, as in many social and cultural pursuits. Therefore, earlier agentic options will remain feasible and often desirable, as in matters of faith and family. But they will exhibit reduced functionality, compared to fully digitalized, generative options. In fact, engineers plan for these options too, recognizing that people will sometimes wish to control technological functioning for recreational or other reasons (Simmler \& Frischknecht, 2021). However, extra problems arise when agents adopt different metamodels at the same time. I will return to this topic in later sections.

In the meantime, as the preceding argument explains, a central feature of any agentic metamodel is the quality of its supervision. That is, how and to what degree, the metamodel is copied or composed, self-regulated or externally controlled, and from which source. We can therefore distinguish metamodels in terms of their supervision, and particularly, in terms of human and technological sources of supervision. Nine alternative patterns are depicted in Fig. 2.6. On the horizontal dimension, the figure 
shows the level of human supervision. While the vertical dimension shows the level of technological supervision. Each segment of Fig. 2.6 therefore shows two potential sources and three levels of supervision of agency, low, medium, and high. Circles with dashed borders represent technological supervision, while human supervision is represented by circles with solid borders. The circles in each segment overlap because both types of supervision interact. It is important to note, that the size of these shapes does not represent the absolute strength or complexity of supervision, but rather their relative significance in any metamodel.

First, consider segment 1 in Fig. 2.6. It shows the type of simple supervision in replicative metamodels of agency, which dominated during premodernity. Human supervision is shown by the small circle with a solid border, and technological supervision by the small circle with a dashed border. In this metamodel, therefore, technological and human levels of supervision are both low. Supervision is routine, encoded, and replicative, relying on communal rituals, perhaps simple tools for writing,

Human Supervision

Low

Medium

High

Technological

Supervision

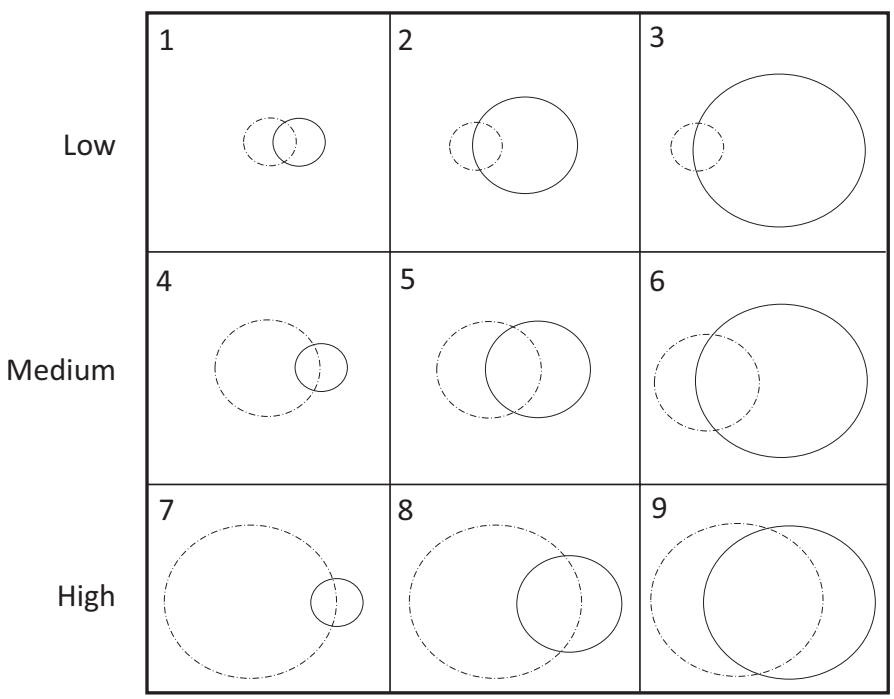

Fig. 2.6 Historical eras of agentic supervision 
counting, and communicating, but not much more. In summary, replicative metamodels of agency have relatively low levels of autonomous, technological, and human supervision. The metamodels offer few degrees of freedom. In this regard, segment 1 complements the premodern problematics depicted earlier, by the segments in Fig. 2.5 which have only one functional dot.

Now consider segments 2, 4, and 5 as well. They show metamodels with greater technological capabilities, as in modernity. In fact, segments $1,2,4$, and 5, represent the patterns of supervision in modern, adaptive metamodels of agency. They complement the earlier depiction of modern problematics in Fig. 2.5, and especially the role of agent X in the collective. Most notably, there are now four options in Fig. 2.6, combining medium or low human supervision, with medium or low technological supervision. In other words, both human and technological supervision have advanced. Relevant technologies are largely mechanical and analogue and provide a moderate assistance to the supervision of agency. Human capabilities also advance, at least for some people, but still within constraints. Indeed, the limits of human supervisory capability are a persistent theme of modernity. Hence, the metamodels of agency represented by segments 1, 2, 4, and 5 in Fig. 2.6 bestow greater degrees of freedom, compared to the preceding replicative option.

Regarding the details, segment 5 shows medium levels of human and technological supervision. This encompasses technologically assisted domains, such as surgical practice, in which human and technological supervision are both critical. Segment 2, on the other hand, shows dominant human supervision, similarly to premodern contexts. Segment 4 shows the opposite scenario, in which technological supervision dominates, as it often does in mechanical systems which operate independently of human intervention. Many automated processes are like this. In summary, modernity exhibits different levels of human and technological supervision, generating alternative agentic options. For this reason, modernity also presents more frequent choices and dilemmas, about which metamodel of agency fits best, when, and why.

Next, by including all 9 segments of Fig. 2.6, we have an illustration of digitalized, generative metamodels of agency. Clearly, there are more feasible metamodels and choices. Human capabilities are more developed in 
segments 3, 6, and 9, as are technological capabilities in segments 7, 8, and 9. Moreover, augmented agents can exploit all these metamodels, and potentially in real time. This dynamism will be significantly owing to the entrogenous mediation mechanisms discussed previously and illustrated in Fig. 2.4. But these mechanisms also bring new challenges and risks. If supervision is poor, agents might develop in overly divergent or convergent ways and become dysfunctional, for example, adopting the option in segment 3 when the balanced option in segment 5 is more appropriate. In this respect, the whole of Fig. 2.6 captures a central challenge for digitally augmented agents, namely, the complexity of supervising human-machine collaboration (see Murray et al., 2020; Simmler \& Frischknecht, 2021).

Depending on the context, therefore, each metamodel in 2.6 can be effective and appropriate. To begin with, scenario 1 will be largely routine. Whereas segment 9 shows the opposite scenario. Human and technological supervision are both strong and assertive. This metamodel is best suited to highly digitalized, complex, dynamic contexts. Expert medical practice is a good example, in which artificial and human agents both supervise critical aspects of collaborative functioning. The major risk is conflict within the augmented agent, for example, when the circles in segment 9 overlap less and supervision is poorly coordinated. Other scenarios show the alternatives in-between, combining low, medium, and high levels of supervision. Sometimes human supervision is clearly dominant, as in scenario 3. This metamodel will be fully humanized and supervision will be guided by ordinary values and commitments. However, the risk is that human myopia and bias will intrude and distort the system. Next, there are metamodels in which technological supervision is dominant, as in scenario 7. These are highly digitalized, but the risk is that human inputs are excluded inappropriately. People could become digitally docile and overly dependent. Segment 5 is intermediate. It includes moderate supervision of both kinds, but significant freedom as well. This metamodel could be appropriate in exploratory, creative contexts. The risk is that agents may lack enough supervision and tend toward incoherence.

Furthermore, segment 1 in a digitalized world, has the same general pattern of supervision in a premodern context. In other words, the type of routine agency which dominated during premodernity may still occur 
within a digitalized world. Even in the period of digitalization, that is, people may adopt purely replicative models of agency, over digitally augmented options. This may seem counterintuitive, but in fact, it will be widespread. Earlier, I cited traditional cultural and faith traditions as examples of such choices. However, this might lead to the reinforcement of human myopias and biases. Next, segments 1,2, 4, and 5 are equivalent in the adaptive and generative systems. What this means, is that the modern patterns of supervision can also occur in a digitalized world. People will still exhibit adaptive, modern approaches, and eschew high levels of digital augmentation. For example, purely human inputs and adaptive learning will likely remain dominant in some social and professional contexts. Moreover, such metamodels may be fully functional, assuming the choice of metamodel fits the context. The major challenge in all scenarios, therefore, is to develop mutual understanding, trust, and empathy, within the augmented agent.

\subsection{Implications for Augmented Humanity}

Significant dilemmas therefore confront digitally augmented humanity, conceived in terms of purposive agency, primarily because human and artificial agents have different capabilities and potentialities. Compared to artificial agents, humans are often myopic, sluggish, layered, and approximating. While relative to humans, artificial agents are increasingly expansive, fast, compressed, and precise. When combined in augmented collaboration, these divergent characteristics are either complementary or conflicting. If the collaboration is well supervised, they are complementary. Human and artificial agents strengthen each other and mitigate the other's limitations. However, if poorly supervised, they are conflicting, and combination leads to poorly fitting metamodels: either underfitting, meaning metamodels admit too much noise and variance, and fail to clarify potential models of interest; or overfitting, meaning they omit too much noise and potential variance, thereby excluding potential models of interest. Metamodels of agency can therefore skew inappropriately, either amplifying human priors, especially myopias and biases. Or they skew the other way, by amplifying digital processes which 
diminish and override the human. Sometimes both patterns of distortion will occur, resulting in extremely divergent systems. In each scenario, augmented agents will underperform and incur functional losses. These risks already attract significant attention from computer scientists (Gavrilov et al., 2018). Moving forward, they will be major concerns for human scientists as well. The supervision of augmented agency will require new theory and techniques.

\section{Implications for Specific Domains}

Hence, the following challenge arises for theory and practice: to understand which aspects of human and artificial supervision should be reinforced, adapted, or relaxed, so that augmented agents maximize the benefits of digitalization, while preserving core values, commitments, and other humanistic qualities. Important legacies are at stake. Many aspects of modernity, and even premodernity, could remain fulfilling and functional, even in a highly augmented world, assuming agents adopt these options appropriately and avoid superstitious thinking and distorting priors. For example, people will continue to find meaning in religious narrative and spiritual commitment, and purely human supervision could be fully appropriate in everyday life. Augmented humanity will benefit by preserving these options. The challenge is dynamically to determine which values and commitments are humanizing, and which are distorting, when and why, and then to supervise them effectively (Sen, 2018).

Collaborative supervision is therefore a major challenge for augmented humanity. It reflects the core problematic of digitalization: how to combine and balance human and artificial capabilities and potentialities? It will impact all aspects of agentic form and function. The following chapters examine important areas of impact. In doing so, they will emphasize different aspects of the generative agentic metamodel in Fig. 2.3:

- Chapter 3 is about agentic modality, which involves the overall metamodeling of individual, group, and collective form and function.

- Chapter 4 examines problem-solving, which involves sensoryperceptive (SP) inputs about problems, and cognitive-affective processing (CA) then searches for solutions. 
- Chapter 5 examines cognitive empathy for other minds, which takes sensory-perceptive (SP) inputs about other minds, and cognitiveaffective processing (CA) then searches for empathic solutions.

- Chapter 6 focuses on self-regulation, which is a major cognitiveaffective processing unit (PU) and integral to action generation (AG), among other reflexive functions.

- Chapter 7 is about evaluation of performance (EP), which assesses the outcomes of behavior performances, referencing some criteria.

- Chapter 8 examines learning, especially in which evaluation of performance leads to feedforward (FF) and feedback (FB) updates.

- Chapter 9 is about self-generation, that is, how augmented agents choose and transform their own metamodels and narratives.

- Chapter 10 summarizes the argument and looks forward to a science of digitally augmented agency.

\section{Reality and Truth}

Additional consequences follow for the core criteria of reality, truth, and ethics, or in other words, for core ontological, epistemological, ethical, and cultural commitments. To begin with, recall that dominant agentic metamodels at any time, tend to reflect the limitations of capability and potentiality. From this perspective, ideal criteria are extrapolations of human limitation (Appiah, 2017). As an adaptive mechanism, such idealization is explicable and often functional. It bolsters agents' self-efficacy and sense of security because human capabilities appear to encompass the limits of reality, truth, and value. Ideals also provide predictable meaning and guidance. Of course, empirical science consistently exposes the contingency of such ideals, which partly explains why scientific advance is often controversial. It challenges inflated self-efficacy and identity. Ironically, therefore, the greater the impact of applied science, including digitalization, the more resistance it may provoke.

To illustrate, consider the contemporary period. In much modern thinking, material nature is opposed to conscious mind. Kant (1998) clearly established the distinction: pure abstract reasoning draws from 
ideal spirit and is categorically different from practical thought in the temporal realm. Rational mind was a category of immaterial reality, while practical reasoning translated mind into the contingent world. From a purely functional perspective, this belief liberates autonomous mind from premodern myth and superstition, while protecting it from mechanistic reduction. In addition, by separating mind and nature, it provides a rationale for human exploration and exploitation of the natural world. However, in the light of artificial intelligence and cognitive neuroscience, many now question these assumptions. They rightly observe that artificial intelligence blends cognitive and material phenomena, while neuroscience promises to explain consciousness in terms of natural and ecological mechanisms (Seth, 2018).

Furthermore, as Chap. 1 explains, the insights of digitally augmented neuroscience imply that the fundamental hyperparameters of mind and consciousness are not directly accessible to reflexive consciousness itself. Instead, researchers use neurophysiological and digital techniques. In fact, advanced artificial intelligence already simulates significant aspects of conscious mental life, including calculative and associative reasoning, intuition and increasingly, empathy and personality (Mehta et al., 2019). As these technologies mature, traditional distinctions between mind and nature, and between virtual and material, will appear increasingly contingent, more functional than fundamental. This shift is foreshadowed by the generative agentic metamodel in Fig. 2.3. It shows that digitalized processes infuse all areas of agentic functioning.

\section{Value and Commitment}

Earlier in this chapter, I also noted that ancient thought remains relevant today. For example, the ancients examined the hedonic nature of life, contrasting pleasure and pain, gain and loss, life and death. Not surprisingly, humans approach the former conditions and try to avoid the latter. These hedonic principles still run deep in Western thinking. To illustrate, Higgins' (1998) Regulatory Focus Theory is explicitly hedonic. It contrasts the prevention of pain and loss, against the promotion of pleasure and gain. As another example, Kahneman and Tversky's (2000) Prospect 
Theory draws fundamental distinctions between potential gains and losses in behavioral decision-making. And to be sure, life was and is, deeply hedonic. Human beings naturally seek to avoid pain, loss, and death, while hoping for pleasure, gains, and life.

Going further, however, the ancients also thought about eudaimonic needs, or the human desire for overall well-being and to live a good life (Aristotle, 1980). These aspirations transcend the hedonic avoidance of loss and pursuit of gains. Rather, eudaimonia embraces the whole of experience, and hence, the totality of human purpose and potentiality (Di Fabio \& Palazzeschi, 2015). Notably, this concept has gained fresh prominence in contemporary thought, including positive psychology (Seligman \& Csikszentmihalyi, 2000), new thinking in economics by Amartya Sen (2004) and others, and value-based models of social organization (Lounsbury \& Beckman, 2015).

This conceptual shift may be partly explained by the rapid growth of capabilities and potentialities brought about by modernity and its related changes. Put simply, flourishing is now more feasible for more people (Phelps, 2013). Of course, widespread discrimination, deprivation, and inequality persist, and new divisions have emerged. Nonetheless, owing in part to digital augmentation, the realm of agentic potentiality is expanding. Human beings will be able to curate new metamodels of being and becoming, including dynamic compositions of the self and community. Agentic potentiality will be vastly different in such a world. In fact, this transformation will likely spawn a new science of eudaimonics, integrating value commitments broadly conceived, where commitment in this context is defined as being dedicated, feeling obligated and bound, to some value, belief, or pattern of action. Such a science would complement the existing disciplines of economics, ethics, politics, and aesthetics (see Di Fabio \& Palazzeschi, 2015; Sen, 2004). I will return to this possibility in the final chapter.

In the meantime, advances in cognitive neuroscience and computer science reinforce the fact that fundamental properties of mind and consciousness cannot be accessed via ordinary means (Seth, 2018). Introspection and intersubjectivity are no longer enough, nor the anthropomorphic conceptions which these methods support. New concepts 
and techniques are required. Yet at the same time, human beings live in and through ordinary consciousness. It is fundamental to being and remaining human. This presents a core challenge for augmented agency, which is to maintain the value and significance of consciousness and mental life, even as science frees itself from anthropomorphic constraints. In fact, this dilemma reinforces the role of commitments in the supervision of augmented agency, because commitments will anchor agents in lived experience. Commitments validate and sustain ordinary consciousness and mind, without claiming scientific status. They are simply and importantly human. Hence, commitments will play a central role in the supervision of augmented agency. They will reinforce humanistic values, helping to preserve the experience of ordinary mind and consciousness in a digitalized world.

\section{References}

Appiah, K. A. (2017). As if: Idealization and ideals. Harvard University Press. Aristotle. (1980). The Nicomachean Ethics (D. Ross, Trans.). Oxford University Press.

Baldwin, C. Y. (2008). Where do transactions come from? Modularity, transactions, and the boundaries of firms. Industrial \& Corporate Change, 17(1), 155-195.

Baldwin, C. Y. (2018). Design rules, volume 2: How technology shapes organizations; chapter 6 the value structure of technologies, part 1: Mapping functional relationships. Harvard Business School, Harvard Business School Research Paper Series (19-037).

Bandura, A. (2007). Reflections on an agentic theory of human behavior. Tidsskrift-Norsk Psykologforening, 44(8), 995.

Basso, D., \& Belardinelli, M. O. (2006). The role of the feedforward paradigm in cognitive psychology. Cognitive Processing, 7(2), 73-88.

Caro, M. F., Josyula, D. P., Cox, M. T., \& Jimenez, J. A. (2014). Design and validation of a metamodel for metacognition support in artificial intelligent systems. Biologically Inspired Cognitive Architectures, 9, 82-104. 
Clune, J., Pennock, R. T., Ofria, C., \& Lenski, R. E. (2012). Ontogeny tends to recapitulate phylogeny in digital organisms. The American Naturalist, 180(3), E54-E63.

Di Fabio, A., \& Palazzeschi, L. (2015). Hedonic and eudaimonic well-being: The role of resilience beyond fluid intelligence and personality traits. Frontiers in Psychology, 6, 1367.

Dong, H., Ma, W., Wu, Y., Zhang, J., \& Jiao, L. (2020). Self-supervised representation learning for remote sensing image change detection based on temporal prediction. Remote Sensing, 12(11), 1868.

Feurer, M., \& Hutter, F. (2019). Hyperparameter optimization. In Automated machine learning (pp. 3-33). Springer.

Fiedler, K., \& Juslin, P. (Eds.). (2006). Information sampling and adaptive cognition. Cambridge University Press.

Fiedler, K., \& Wanke, M. (2009). The cognitive-ecological approach to rationality in social psychology. Social Cognition, 27(5), 699-732.

Gavrilov, A. D., Jordache, A., Vasdani, M., \& Deng, J. (2018). Preventing model overfitting and underfitting in convolutional neural networks. International Journal of Software Science and Computational Intelligence (IJSSCI), 10(4), 19-28.

Geertz, C. (2001). Available light. Princeton University Press.

Giddens, A. (1991). Modernity and self-identity: Self and society in the late modern age. Stanford University Press.

Hackforth, R. (1972). Plato: Phaedo. Cambridge University Press.

Heaven, D. (2020, January 21). IBM's debating ai just got a lot closer to being a useful tool. MIT Technology Review.

Hegel, G. W. F. (1980). Lectures on the philosophy of world history. Cambridge University Press.

Higgins, E. T. (1998). Promotion and prevention: Regulatory focus as a motivational principle. Advances in Experimental Social Psychology, 30, 1-46.

Howell, B. M. (2019). Managing emerging technology and organizations with agility. In Advances in the technology of managing people: Contemporary issues in business. Emerald Publishing Limited.

Ibarra, H., \& Obodaru, O. (2016). Betwixt and between identities: Liminal experience in contemporary careers. Research in Organizational Behavior, $36,47-64$.

Isin, E. F. (2002). Being political: Genealogies of citizenship. University of Minnesota Press. 
Kahneman, D., \& Tversky, A. (2000). Prospect theory: An analysis of decision under risk. In D. Kahneman \& A. Tversky (Eds.), Choices, values, and frames (pp. 17-43). Cambridge University Press.

Kant, I. (1998). Critique of pure reason (P. Guyer \& A. W. Wood, Trans.). Cambridge University Press.

Kaul, P., \& Lall, B. (2019). Riemannian curvature of deep neural networks. IEEE Transactions on Neural Networks and Learning Systems, 31(4), 1410-1416.

Kempis, T. à. (1952). The imitation of Christ (c. 1420) (L. Sherley-Price, Trans.). London: Penguin Classics.

Kirk, G. S. (1954). Heraclitus: The cosmic fragments. Cambridge University Press. Kouvaris, K., Clune, J., Kounios, L., Brede, M., \& Watson, R. A. (2015). How evolution learns to generalise: Principles of under-fitting, over-fitting and induction in the evolution of developmental organisation. arXiv preprint arXiv:1508.06854.

Lee, S. H., \& Ro, Y. M. (2015). Partial matching of facial expression sequence using over-complete transition dictionary for emotion recognition. IEEE Transactions on Affective Computing, 7(4), 389-408.

Locke, J. (1967). Locke: Two treatises of government. Cambridge University Press. Lounsbury, M., \& Beckman, C. M. (2015). Celebrating organization theory. Journal of Management Studies, 52(2), 288-308.

March, J. G. (2010). The ambiguities of experience. Cornell University Press.

Mayr, E. (2002). What evolution is. Weidenfeld \& Nicolson.

Mehta, Y., Majumder, N., Gelbukh, A., \& Cambria, E. (2019). Recent trends in deep learning based personality detection. Artificial Intelligence Review, 1-27.

Mischel, W., \& Shoda, Y. (1995). A cognitive-affective system theory of personality: Reconceptualizing situations, dispositions, dynamics, and invariance in personality structure. Psychological Review, 102(2), 246-268.

Mischel, W., \& Shoda, Y. (1998). Reconciling processing dynamics and personality dispositions. Annual Review of Psychology, 49(1), 229-258.

Murray, A., Rhymer, J., \& Sirmon, D. G. (2020). Humans and technology: Forms of conjoined agency in organizations. Academy of Management Review (online).

Norvig, P., \& Russell, S. (2010). Artificial intelligence: A modern approach (3rd ed.). Pearson. 
O’Reilly, C. A., \& Tushman, M. L. (2013). Organizational ambidexterity: Past, present, and future. The Academy of Management Perspectives, 27(4), 324-338.

Ojha, V. K., Abraham, A., \& Snášel, V. (2017). Metaheuristic design of feedforward neural networks: A review of two decades of research. Engineering Applications of Artificial Intelligence, 60, 97-116.

Phelps, E. S. (2013). Mass flourishing: How grassroots innovation created jobs, challenge, and change. Princeton University Press.

Pinker, S. (2010). The language instinct: How the mind creates language. HarperCollins.

Pinker, S. (2018). Enlightenment now: The case for reason, science, humanism, and progress. Penguin.

Reill, P. H. (2005). Vitalizing nature in the enlightenment. University of California Press.

Seligman, M. E. P., \& Csikszentmihalyi, M. (2000). Positive psychology: An introduction. American Psychologist, 55(1), 5-14.

Sen, A. (2004). Economic methodology: Heterogeneity and relevance. Social Research, 71(3), 583-614.

Sen, A. (2018). The importance of incompleteness. International Journal of Economic Theory, 14(1), 9-20.

Seth, A. K. (2018). Consciousness: The last 50 years (and the next). Brain and Neuroscience Advances, 2, 1-6.

Shalev-Shwartz, S., Shammah, S., \& Shashua, A. (2017). On a formal model of safe and scalable self-driving cars. arXiv preprint arXiv:1708.06374.

Shoda, Y., LeeTiernan, S., \& Mischel, W. (2002). Personality as a dynamical system: Emergence of stability and distinctiveness from intra- and interpersonal interactions. Personality and Social Psychology Review, 6(4), 316-325.

Simmler, M., \& Frischknecht, R. (2021). A taxonomy of human-machine collaboration: Capturing automation and technical autonomy. AI \& SOCIETY, 36(1), 239-250.

Simon, H. A. (1996). The sciences of the artificial (3rd ed.). The MIT Press.

Sorabji, R. (2006). Self: Ancient and modern insights about individuality, life, and death. OUP.

Spar, D. L. (2020). Work mate marry love: How machines shape our human destiny. Farrar, Straus and Giroux.

Thaler, R. H. (2016). Behavioral economics: Past, present, and future. American Economic Review, 106(7), 1577-1600. 
Vilela, M., \& Hochberg, L. R. (2020). Applications of brain-computer interfaces to the control of robotic and prosthetic arms. In Handbook of clinical neurology (Vol. 168, pp. 87-99). Elsevier.

Walker, J. (2000). Rhetoric and poetics in antiquity. Oxford University Press.

Williams, B. (1993). Shame and necessity. University of California Press.

Wykowska, A. (2021). Robots as mirrors of the human mind. Current Directions in Psychological Science, 30(1), 34-40.

Open Access This chapter is licensed under the terms of the Creative Commons Attribution 4.0 International License (http://creativecommons.org/licenses/ by/4.0/), which permits use, sharing, adaptation, distribution and reproduction in any medium or format, as long as you give appropriate credit to the original author(s) and the source, provide a link to the Creative Commons licence and indicate if changes were made.

The images or other third party material in this chapter are included in the chapter's Creative Commons licence, unless indicated otherwise in a credit line to the material. If material is not included in the chapter's Creative Commons licence and your intended use is not permitted by statutory regulation or exceeds the permitted use, you will need to obtain permission directly from the copyright holder.

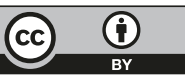

